\title{
INCLUSÕES NÃO METÁLICAS NO AÇO INOXIDÁVEL FERRÍTICO COM ADIÇÕES DE TITÂNIO E CÁLCIO NO FORNO PANELA*
}

\section{Resumo}

Márcio Nascimento Cunha ${ }^{1}$ Valdeci Paula Alvarenga ${ }^{2}$ Hélio José Batista Alves ${ }^{3}$

Titânio é adicionado aos aços inoxidáveis ferríticos para melhorar a resistência à corrosão e estabilizar a estrutura ferrítica. Devido à afinidade com oxigênio no aço líquido, perdas de titânio por oxidação são comuns, sendo as adições realizadas após etapas de desoxidação. Inclusões de titânio podem causar obstruções na válvula submersa durante o Lingotamento e também defeitos superficiais no produto final. Técnicas para obter inclusões menos prejudiciais às propriedades dos aços têm sido empregadas e a obtenção de inclusões com ponto de fusão mais baixo após tratamento com cálcio tem se mostrado efetivo. Os objetivos deste trabalho foram avaliar a composição química das inclusões no aço inoxidável 409 após adições de titânio no Forno Panela e o efeito da adição de cálcio no comportamento das inclusões. Amostras do aço líquido foram retiradas no Forno Panela em corridas com e sem adição de Ca-Si. A composição química das inclusões foi analisada no MEV-EDS.

Palavras-chave: Aço inoxidável 409; Inclusões; Óxidos; Forno panela.

\section{NON METALLIC INCLUSIONS IN FERRITIC STAINLESS STEEL WITH TITANIUM AND CALCIUM ADITIONS IN THE LADLE FURNACE}

\section{Abstract}

Titanium is added in ferritic stainless steels to improve corrosion resistance and to stabilize the ferritic structure. Titanium losses by oxidation are also common due to the oxygen affinity in the molten steel. Inclusions formed by titanium oxidation can cause nozzle clogging and defects in the final product. Inclusions with lower melting point have showed less detrimental to the steel properties and it has been reached with calcium treatment. This work had the objective to evaluate the chemical composition of the inclusions on the stainless steel type 409 after titanium additions in the Ladle Furnace and the effect of the calcium addition in the inclusions behavior. Samples of the melt steel were obtained in baths with and without Ca-Si additions in the Ladle Furnace process. The inclusions were analyzed by SEM-EDS.

Keywords: Stainless steel type 409; Inclusions; Oxide; Ladle furnace.

1 Mestre em Engenharia Metalúrgica, Engenheiro Metalurgista do produto inox, Aperam South America, Timóteo, Minas Gerais, Brasil.

2 Mestre em Engenharia Metalúrgica, Engenheiro Metalurgista de processo, Aperam South America, Timóteo, Minas Gerais, Brasil.

3 Mestre em Engenharia Metalúrgica, Pesquisador do produto inox, Aperam South America, Timóteo, Minas Gerais, Brasil.

4 Doutor em Engenharia Metalúrgica, Professor do departamento de Engenharia Metalúrgica e de Materiais da UFMG, Belo Horizonte, Minas Gerais, Brasil. 


\section{INTRODUÇÃO}

Um dos grandes desafios na produção do aço 409 no mundo é o processo de solidificação. A presença de titânio na composição química e a afinidade deste elemento pelo oxigênio tornam o aço susceptível a uma grande quantidade de inclusões devido à formação de óxidos complexos de titânio. Inclusões de titânio passam para o molde através da válvula submersa e geram grande quantidade de defeitos após laminação e, em casos mais graves, causam obstrução da válvula submersa com interrupção do lingotamento [1, 2].

Como não é possível a remoção completa das inclusões formadas nos processos de elaboração do aço líquido, técnicas para obtenção de inclusões menos prejudiciais têm sido empregadas para minimizar os efeitos das inclusões remanescentes. Para isto, o controle da dureza das inclusões tem se mostrado efetivo, visto que inclusões com efeitos deletérios são aquelas mais duras e não são deformáveis [3].

A forma mais efetiva de reduzir a dureza das inclusões é diminuir seu ponto de fusão Em aços desoxidados ao alumínio, a adição de cálcio modifica as inclusões de alumina sólidas nas temperaturas de elaboração do aço líquido para inclusões do tipo aluminato de cálcio, as quais são líquidas [3].

Os objetivos deste trabalho foram avaliar a composição química das inclusões no aço inoxidável 409 após adições de titânio e o efeito da adição de cálcio no comportamento das inclusões.

\section{MATERIAL E MÉTODOS}

\subsection{Amostragem}

Amostras foram retiradas no Forno Panela em uma corrida com processo padrão com adição de titânio e outra com adição titânio e de Ca-Si $(20 \mathrm{Kg})$ na sequencia. A figura 1 representa o fluxo de produção do aço 409 destacando a etapa do Forno Panela.

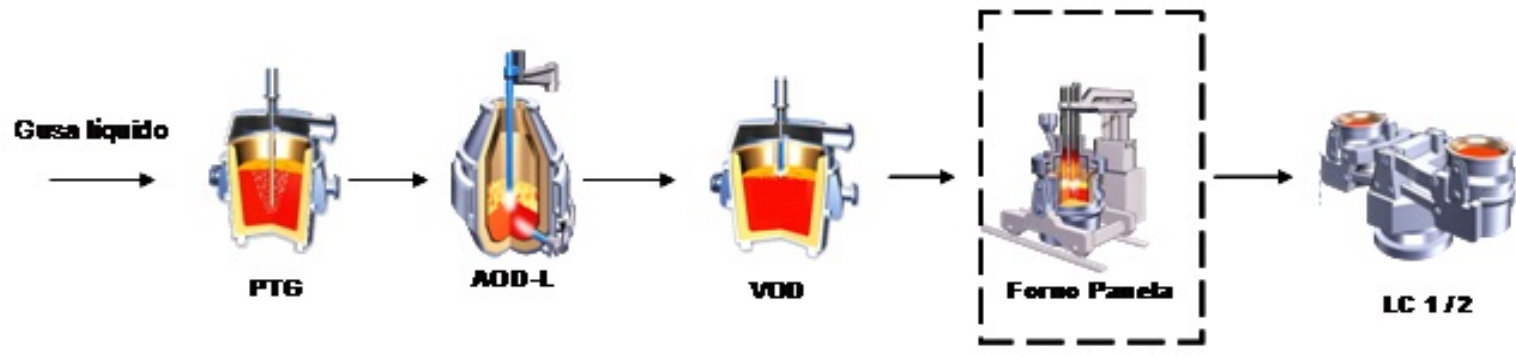

Figura 1. Fluxo de produção do aço 409 destacando a etapa do Forno Panela

O método de amostragem, assim como o tipo da amostra, foi o mesmo adotado para realização de análises químicas durante a produção. A figura 02 mostra o tipo de amostra obtida. 


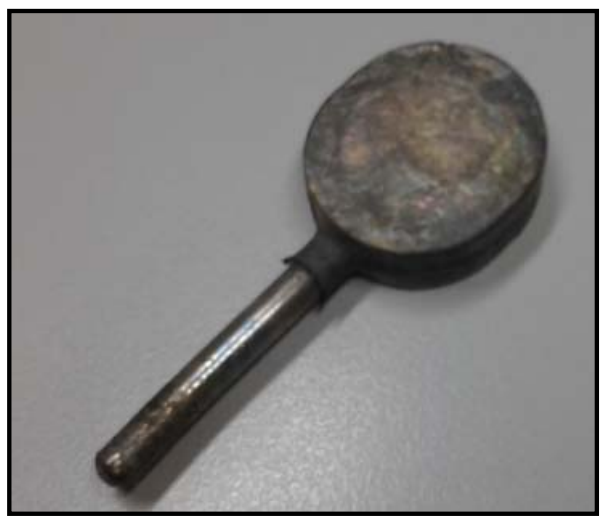

Figura 2. Amostra obtida para análise.

As tabelas 1 e 2 apresentam o esquema de amostragem para corridas com e sem adição de Ca-Si.

Tabela 1. Esquema de amostragem do aço na Aciaria

\begin{tabular}{|c|c|l|l|}
\hline Corrida & Amostra & \multicolumn{1}{|c|}{ Processo } & \multicolumn{1}{c|}{ Momento de amostragem } \\
\hline C1 & A1 & Forno Panela & Após adição de titânio (5 min flotação). \\
\hline C2 & A2 & Forno Panela & Após adição de titânio (5 min flotação). \\
\hline C2 & A3 & Forno Panela & Após adição de Ca-Si. \\
\hline
\end{tabular}

\subsection{Preparação das Amostras}

Amostras para análise microscópica foram preparadas no laboratório do Centro de Pesquisas da APERAM, seguindo as etapas de corte, embutimento, lixamento e polimento metalográfico. Para evitar regiões defeituosas na amostras, o procedimento adotado foi o mesmo sugerido por Cunha et. al. (2015).

\subsection{Análises}

Análises de microscopia eletrônica foram realizadas no Centro de Pesquisas da APERAM. Foram utilizados aumentos 500X, buscando a área com maior quantidade de inclusões (pior área da amostra).

A análise de composição química das inclusões foi realizada com sistema EDS, acoplado a um Microscópio Eletrônico de Varredura (MEV) nas amostras obtidas após adição de titânio e após adição de Ca-Si. A pior área da amostra foi dividida em 50 campos e todas as inclusões nestes campos foram analisadas. Foram considerados os resultados de análises química somente das inclusões com tamanho $\geq 2 \mu \mathrm{m}$.

Para análise nos diagramas de superfícies liquidus dos sistemas de óxidos que representaram as principais inclusões, os valores dos elementos obtidos com EDS foram convertidos em óxidos através de cálculos estequiométricos simples conforme proposto por Bielefeldt (2005). Em alguns casos, o percentual em massa dos óxidos foi convertido para fração molar. 


\section{RESULTADOS E DISCUSSÃO}

\subsection{Composição química das inclusões tipo óxidos para corrida C1.}

No Forno Panela (amostra A1), após adição de titânio na corrida $\mathrm{C} 1$, as principais inclusões encontradas foram óxidos de titânio com cálcio, magnésio e alumínio como mostra a tabela 2.

Tabela 2. Inclusões no Forno Panela após adição de titânio.

\begin{tabular}{|c|c|c|c|c|}
\hline Composição Química & $\begin{array}{c}\text { Inclusão 1 } \\
\text { "Tipo Óxido" }\end{array}$ & $\begin{array}{c}\text { Inclusão 2 } \\
\text { "Tipo Óxido" }\end{array}$ & $\begin{array}{c}\text { Inclusão 3 } \\
\text { "Tipo Óxido" }\end{array}$ & $\begin{array}{c}\text { Inclusão 4 } \\
\text { "Tipo Óxido" }\end{array}$ \\
\hline $\mathrm{Ti}$ & $40 \%$ & $45 \%$ & $44 \%$ & $60 \%$ \\
\hline $\mathbf{C a}$ & $10 \%$ & $10 \%$ & $15 \%$ & - \\
\hline $\mathbf{A l}$ & $3 \%$ & - & $5 \%$ & - \\
\hline $\mathbf{M g}$ & $10 \%$ & $8 \%$ & - & - \\
\hline $\mathbf{O}$ & $37 \%$ & $37 \%$ & $36 \%$ & $40 \%$ \\
\hline
\end{tabular}

Cunha et. al. (2015) detalha a formação destas inclusões nesta etapa do processo. No entanto, outros autores afirmam que cálcio e magnésio têm origem na escória e posteriormente modificam as inclusões de titânio.

O magnésio dos refratários pode incorporar ao aço líquido de acordo com o mecanismo proposto por Brabie (1996). Além do mais, uma escória rica em $\mathrm{CaO}$ pode fornecer cálcio para a inclusão.

SEO et. al. (2010), mostrou que a presença de titânio no aço pode causar dissolução de magnésio do $\mathrm{MgO}$ ou $\mathrm{CaO}$ contidos nos refratários ou na escória fornecendo $\mathrm{Mg}$ e Ca para as inclusões. O alumínio incorporado proveniente da liga de Fe-Ti reduz a sílica formando inclusões do tipo $\mathrm{Al}_{2} \mathrm{O}_{3}$.

Estas inclusões são representadas pelos sistemas $\mathrm{Al}_{2} \mathrm{O}_{3}-\mathrm{CaO}-\mathrm{TiO}_{2}$ e $\mathrm{MgO}-$ $\mathrm{CaO}-\mathrm{TiO}_{2}$. A tabela 3 apresenta a composição química média das principais inclusões encontradas nesta etapa após a conversão para seus respectivos óxidos.

Tabela 3. Composição química dos óxidos nas inclusões.

\begin{tabular}{|l|l|l|l|}
\hline \multicolumn{2}{|l|}{ Sistema $\mathrm{Al}_{2} \mathrm{O}_{3}-\mathrm{CaO}-\mathrm{TiO}_{2}$} & \multicolumn{3}{|l|}{ Sistema } & $\mathbf{M g O}-\mathrm{CaO}-\mathrm{TiO}_{2}$ \\
\hline $\mathrm{Al}_{2} \mathrm{O}_{3}$ & $11 \%$ & $\mathrm{MgO}$ & $15 \%(0,25$ molar $)$ \\
\hline $\mathrm{CaO}$ & $16 \%$ & $\mathrm{CaO}$ & $17 \%(0,20$ molar $)$ \\
\hline $\mathrm{TiO}_{2}$ & $73 \%$ & $\mathrm{TiO}_{2}$ & $68 \%(0,55 \mathrm{molar})$ \\
\hline
\end{tabular}

As figuras $3 \mathrm{a}$ e $3 \mathrm{~b}$ apresentam os diagramas de superfície liquidus para estes óxidos. Para interpretação da figura $3 b$, a composição química dos óxidos foi convertida para fração molar conforme tabela 3. 

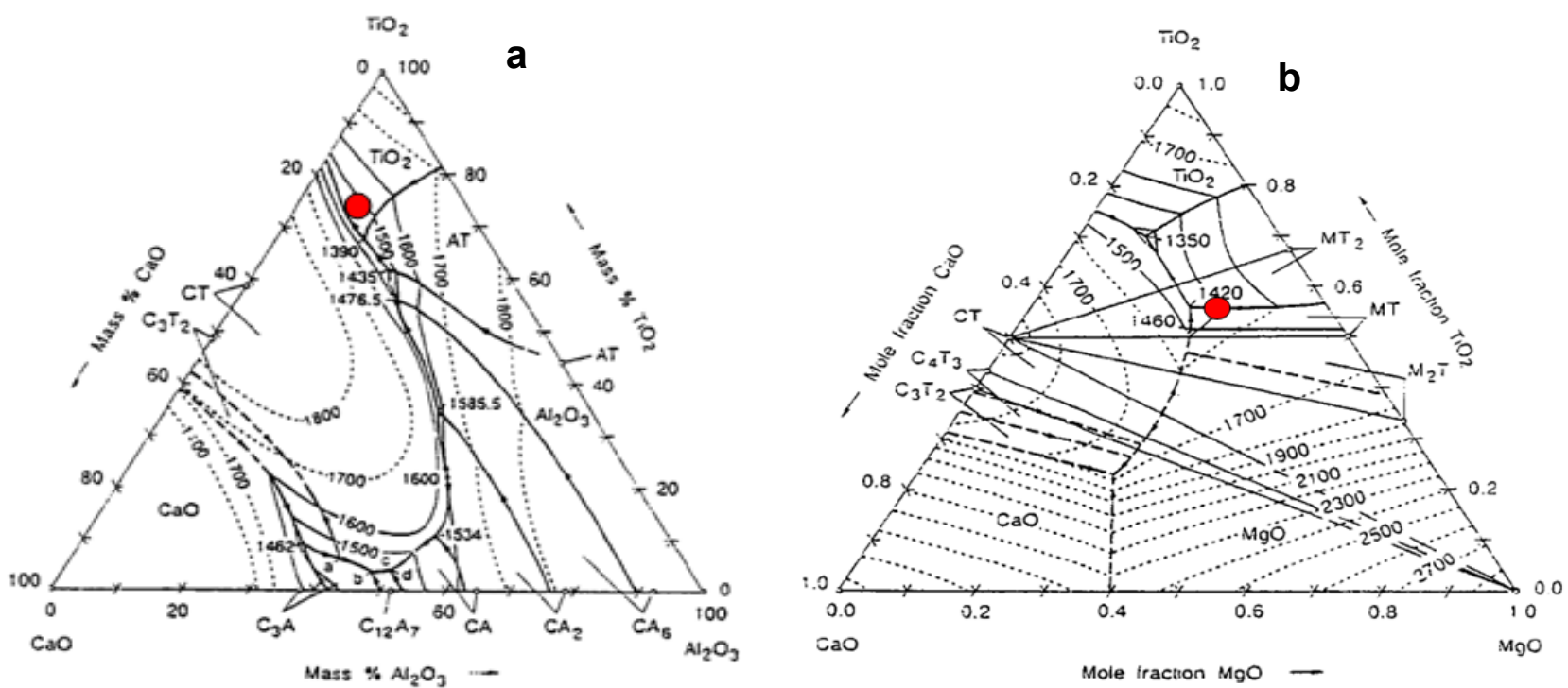

Figura 3. Diagramas de superfícies liquidus. (a) Sistema $\mathrm{Al}_{2} \mathrm{O}_{3}-\mathrm{CaO}-\mathrm{TiO}_{2}$;

(b) $\mathrm{CaO}-\mathrm{MgO}-\mathrm{TiO}_{2}[8]$.

Nesta etapa, inclusões do sistema $\mathrm{Al}_{2} \mathrm{O}_{3}-\mathrm{CaO}-\mathrm{TiO}_{2}$ apresentam temperatura de liquidus de aproximadamente a $1500^{\circ} \mathrm{C}$ e inclusões do sistema $\mathrm{CaO}-\mathrm{MgO}-\mathrm{TiO}_{2}$ temperatura liquidus de aproximadamente $1420^{\circ} \mathrm{C}$. Em ambos os sistemas, as inclusões são líquidas nas temperaturas de elaboração do aço, o que é benéfico.

Com a ajuda dos diagramas de superfície liquidus da figura $3 a$, é possível observar que para inclusões de titânio onde os teores de $\mathrm{Al}_{2} \mathrm{O}_{3}$ são mais predominantes do que os de $\mathrm{MgO}$ (sistema $\mathrm{Al}_{2} \mathrm{O}_{3}-\mathrm{CaO}-\mathrm{TiO}_{2}$ ), é possível obter inclusões com menor temperatura liquidus aumentando os teores de $\mathrm{CaO}$ e diminuindo $\mathrm{Al}_{2} \mathrm{O}_{3}$ e $\mathrm{TiO}_{2}$. Aumentos no teor de $\mathrm{Al}_{2} \mathrm{O}_{3}$ também sugerem a obtenção de inclusões com pontos de fusão mais altos. Isto reforça a necessidade de controlar a entrada de alumínio no Forno Panela nestes aços.

\subsection{Composição química das inclusões tipo óxidos para corrida C2.}

As inclusões no Forno Panela (amostra A2) após adições de titânio na corrida C2 são predominantemente óxidos de titânio com cálcio, magnésio e alumínio da mesma forma que obtido para corrida C1. No entanto, os teores de cálcio apresentaram-se menores em relação à corrida $\mathrm{C} 1$ (tabela 2) após adição de titânio como pode ser observado na tabela 4.

Tabela 4. Inclusões no Forno Panela após adição de titânio.

\begin{tabular}{|c|c|c|c|c|}
\hline Composição Química & $\begin{array}{c}\text { Inclusão 1 } \\
\text { "Tipo Óxido" }\end{array}$ & $\begin{array}{c}\text { Inclusão 2 } \\
\text { "Tipo Óxido" }\end{array}$ & $\begin{array}{c}\text { Inclusão 3 } \\
\text { "Tipo Óxido" }\end{array}$ & $\begin{array}{c}\text { Inclusão 4 } \\
\text { "Tipo Óxido" }\end{array}$ \\
\hline $\mathbf{T i}$ & $60 \%$ & $35 \%$ & $62 \%$ & $70 \%$ \\
\hline $\mathbf{C a}$ & $3 \%$ & $3 \%$ & - & - \\
\hline $\mathbf{A l}$ & - & $9 \%$ & - & - \\
\hline $\mathbf{M g}$ & - & $8 \%$ & - & - \\
\hline $\mathbf{O}$ & $37 \%$ & $37 \%$ & $38 \%$ & $30 \%$ \\
\hline
\end{tabular}


Da mesma forma, na amostra após adição de Ca-Si (amostra A3), os óxidos encontrados foram os mesmos, no entanto houve aumento no teor de Ca conforme tabela 5.

Tabela 5. Inclusões no Forno Panela após adição de Ca-Si.

\begin{tabular}{|c|c|c|c|}
\hline Composição Química & $\begin{array}{c}\text { Inclusão 1 } \\
\text { "Tipo Óxido" }\end{array}$ & $\begin{array}{c}\text { Inclusão 2 } \\
\text { "Tipo Óxido" }\end{array}$ & $\begin{array}{c}\text { Inclusão 3 } \\
\text { "Tipo Óxido" }\end{array}$ \\
\hline $\mathbf{T i}$ & $40 \%$ & $38 \%$ & $67 \%$ \\
\hline $\mathbf{C a}$ & $17 \%$ & - & - \\
\hline $\mathbf{A l}$ & $7 \%$ & $12 \%$ & - \\
\hline $\mathbf{M g}$ & $8 \%$ & $8 \%$ & - \\
\hline $\mathbf{O}$ & $30 \%$ & $42 \%$ & $33 \%$ \\
\hline
\end{tabular}

Estas inclusões também podem ser representadas pelos sistemas $\mathrm{Al}_{2} \mathrm{O}_{3}-\mathrm{CaO}-$ $\mathrm{TiO}_{2}$ e $\mathrm{CaO}-\mathrm{MgO}-\mathrm{TiO}_{2}$. As tabelas 6 e 7 apresentam a composição química média dos óxidos nas inclusões antes e depois da adição de Ca-Si no Forno Panela.

Tabela 6. Composição química dos óxidos nas inclusões (sistema $\mathrm{Al}_{2} \mathrm{O}_{3}-\mathrm{CaO}-\mathrm{TiO}_{2}$ ).

\begin{tabular}{|l|l|l|}
\hline Sistema $\mathrm{Al}_{2} \mathrm{O}_{3}-\mathrm{CaO}-\mathrm{TiO}_{2}$ & Antes da adição de Casi & Após adição de CaSi \\
\hline Oxido & $14 \%$ & $13 \%$ \\
\hline $\mathrm{Al}_{2} \mathrm{O}_{3}$ & $5 \%$ & $23 \%$ \\
\hline $\mathrm{CaO}$ & $81 \%$ & $64 \%$ \\
\hline $\mathrm{TiO}_{2}$ & \\
\hline
\end{tabular}

Tabela 7. Composição química dos óxidos nas inclusões sistema $\left(\mathrm{MgO}-\mathrm{CaO}-\mathrm{TiO}_{2}\right)$.

\begin{tabular}{|l|l|l|}
\hline Sistema $\mathrm{MgO}-\mathrm{CaO}-\mathrm{TiO}_{2}$ & Antes da adição de Casi & Após adição de CaSi \\
\hline Óxido & $13 \%(0,07$ molar $)$ & $11 \%(0,18$ molar $)$ \\
\hline $\mathrm{MgO}$ & $5 \%(0,22$ molar $)$ & $23 \%(0,28$ molar $)$ \\
\hline $\mathrm{CaO}$ & $83 \%(0,71$ molar $)$ & $66 \%(0,54$ molar $)$ \\
\hline $\mathrm{TiO}_{2}$ & & \\
\hline
\end{tabular}

De forma geral, após a adição de Ca-Si houve aumento no teor de $\mathrm{CaO}$ nas inclusões com redução de $\mathrm{TiO}_{2}$ permanecendo os níveis de $\mathrm{Al}_{2} \mathrm{O}_{3}$ e $\mathrm{MgO}$ praticamente constantes.

O efeito da adição de cálcio em obter inclusões com temperaturas liquidus mais baixas foi avaliado considerando a composição química dos óxidos presentes nas inclusões apresentadas nas tabelas 6 e 7 e os diagramas de superfícies liquidus para estes sistemas de óxidos da figura 4. 

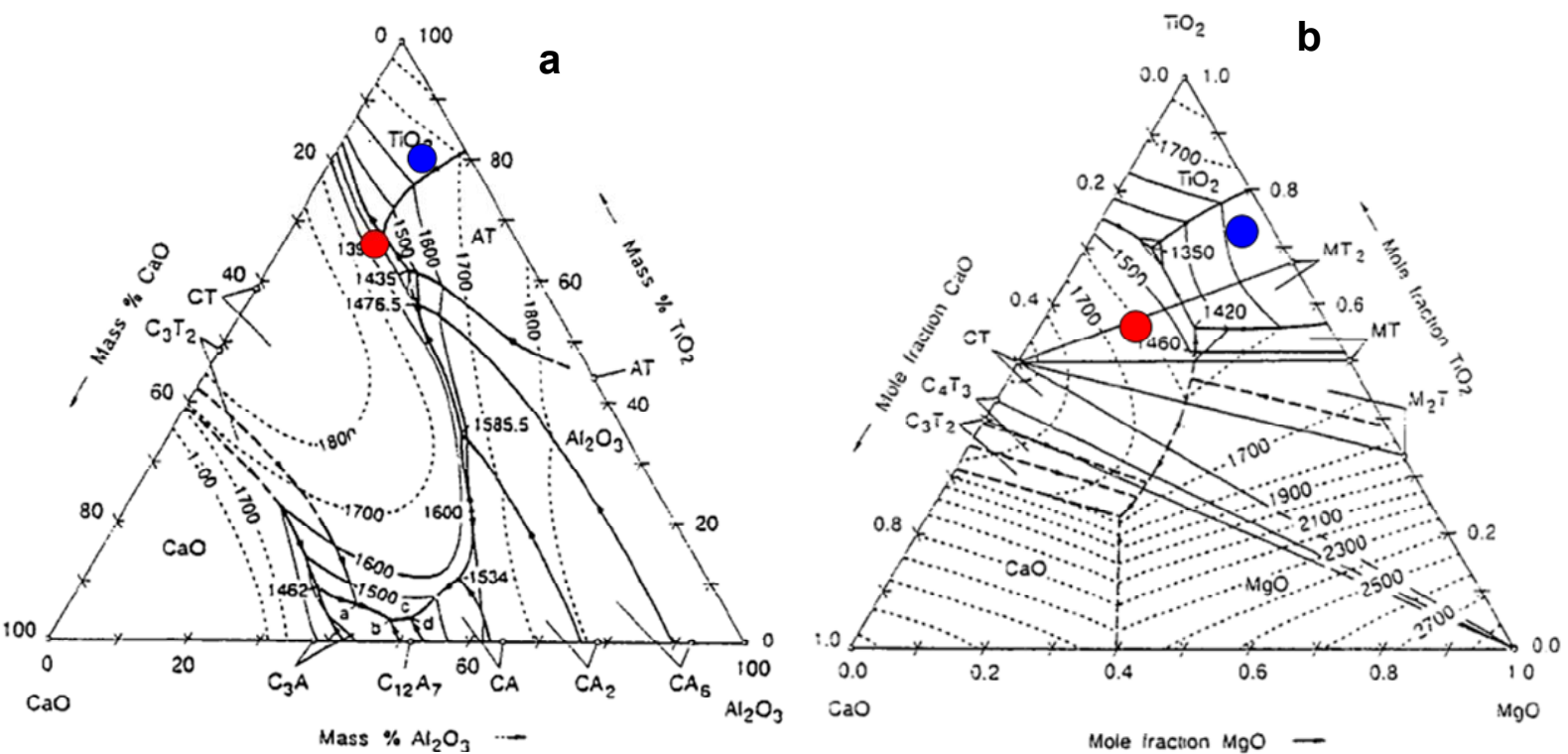

Figura 4. Diagramas de superfícies liquidus destacando o efeito da adição de Ca-Si em modificar a composição química das inclusões. (a) Sistema $\mathrm{Al}_{2} \mathrm{O}_{3}-\mathrm{CaO}-\mathrm{TiO}_{2}$

(b) $\mathrm{CaO}-\mathrm{MgO}-\mathrm{TiO}_{2}[8]$.

A tabela 8 apresenta as temperaturas de liquidus aproximadas, antes e após adição de Ca-Si no Forno Panela obtidas através dos diagramas da figura 4.

Tabela 8. Temperaturas liquidus das inclusões

\begin{tabular}{|l|c|l|c|}
\hline \multicolumn{2}{|l|}{ Sistema $\mathrm{Al}_{2} \mathrm{O}_{3}-\mathrm{CaO}-\mathrm{TiO}_{2}$} & \multicolumn{3}{l|}{ Sistema $\mathbf{M g O}-\mathrm{CaO}-\mathrm{TiO}_{2}$} \\
\hline Condição & Temperatura de liquidus ${ }^{\circ} \mathrm{C}$ & Condição & Temperatura de liquidus ${ }^{\circ} \mathrm{C}$ \\
\hline Antes & 1650 & Antes & 1550 \\
\hline Após & 1435 & Após & 1460 \\
\hline
\end{tabular}

Para ambos os sistemas, a adição de $20 \mathrm{~kg}$ de Ca-Si no Forno Panela após adição de titânio mostrou efeito em modificar as inclusões aumentando o teor de $\mathrm{CaO}$ e deslocando as temperaturas liquidus conforme mostra a figura 4. Antes da adição de $\mathrm{Ca}-\mathrm{Si}$, inclusões com $5 \%$ de $\mathrm{CaO}$ apresentaram como sendo sólidas com temperatura liquidus acima das temperaturas de elaboração do aço 409 que é de $1530^{\circ} \mathrm{C}$ a $1600^{\circ} \mathrm{C}$. Após a adição de $\mathrm{Ca}-\mathrm{Si}$, a temperatura liquidus das inclusões é reduzida com $23 \%$ de $\mathrm{CaO}$, no entanto, para uma região com faixa de temperaturas liquidus muito próxima a $1700^{\circ} \mathrm{C}$, onde as inclusões são totalmente sólidas, o que poder não ser bom tanto para a qualidade quanto para a lingotabilidade do aço.

Estes resultados mostraram que a modificação efetiva de inclusões do tipo $\mathrm{TiO}_{2}$ no aço $409 \mathrm{com}$ tratamento de Ca-Si no Forno Panela depende de pequenas variações no teor de $\mathrm{CaO}$ nas inclusões. Neste caso, o teor de $\mathrm{CaO}$ inicial na inclusão, antes da adição de Ca-Si é um parâmetro importante para definir a quantidade exata a ser adicionada.

$\mathrm{O}$ teor de $\mathrm{CaO}$ nas inclusões pode variar de corrida a corrida, principalmente devido a interação do aço com escórias básicas ricas em com $\mathrm{CaO}$. Como por exemplo, inclusões da corrida C1 no Forno Panela sem adição de $\mathrm{Ca}-\mathrm{Si}$ apresentaram $\mathrm{CaO}$ de $16 \%$ (tabela 3 ). Neste caso, o próprio cálcio fornecido pelo sistema em equilíbrio foi suficiente para obter inclusões benéficas para o aço. Para uma melhor assertividade neste processo, um modelo termodinâmico capaz de 
prever o teor de $\mathrm{CaO}$ na inclusão em função do equilíbrio entre aço, escória e inclusão antes da adição de cálcio pode ser uma solução.

\section{CONCLUSÕES}

No Forno Panela, as principais inclusões encontradas foram óxidos de titânio com alumínio, magnésio e cálcio e apresentaram temperatura liquidus na ordem de $1420^{\circ} \mathrm{C}$ e $1500^{\circ} \mathrm{C}$, ou seja, provavelmente líquidas nas temperaturas de elaboração do aço 409 e benéficas para o processamento e para qualidade.

$\mathrm{O}$ efeito em obter inclusões com baixo ponto de fusão mediante a adição de Ca-Si depende das variações no teor de $\mathrm{CaO}$ na inclusão em uma faixa de temperatura muito estreita de difícil controle. Com pequenas variações, é possível obter tanto inclusões líquidas como sólidas. O teor inicial de $\mathrm{CaO}$ nas inclusões no Forno Panela após adição de titânio e antes da adição de cálcio é um parâmetro importante para definição da quantidade correta de Ca-Si a ser adicionada.

\section{REFERÊNCIAS}

1) KIM, K.H.; DO, K.H.; CHOI, W.J.; LEE, S. B.; KIM, D.S.; PAK, J.J. Inclusion modification by $\mathrm{Al}$ deoxidation and $\mathrm{Ca}$ treatment in Ti containing $18 \% \mathrm{Cr}$ stainless steel melts. Journal of Korean Institute of Metals and Materials. v. 51, №2, pp.113 -118, 2013.

2) SILVA, J.M.F. Melhoria da Lingotabilidade do Aço Inoxidável Ferrítico AISI 409 na ArcelorMittal Inox Brasil. Belo Horizonte: Escola de Engenharia da UFMG, 2009. 74p. (Dissertação, Mestrado em Engenharia Metalúrgica).

3) KANG, Y. B.; CHANG, C.H.; PARK, S.C.; KIM, H.S.; JUNG, I.H; LEE, H.G. Thermodinamics of Inclusions Engineering in Steelmaking and Refining. AIST Transactions. V.3, N 8, p. $153-162,2006$.

4) CUNHA, M. N.; ALVARENGA, V. P.; ALVES, H. J. B. A.; SANTOS, D. B. Análises de inclusões no aço inoxidável ferrítico 409. In: SEMINÁRIO DE ACIARIA, 46, 2015, Rio de Janeiro. Associação Brasileira de Metalurgia, Minas e Materiais (ABM), 2015.

5) BIELEFELDT, W. Estudo do Tratamento com Cálcio na Fabricação do aço SAE 8260. Porto Alegre: Escola de Engenharia, UFRGS, 2005. 133p. (Dissertação, Mestrado em Engenharia Metalúrgica).

6) BRABIE, V. Mechanism of reaction between refractory aluminum deoxidized molten steel. ISIJ International, vol. 36, supplement, 1996, pp. S109-S112.

7) SEO, C.W.; KIM, S.H.; JO, S.K.; SUK, MIN.O. Modification and minimization of spinel $\left(\mathrm{Al}_{2} \mathrm{O}_{3} . \mathrm{xMgO}\right)$ inclusions formed in Ti-added steel melts. Metallurgical and Materials Transactions $B$, p.790-797, 2010.

8) VEREIN DEUTSCHER EISENHUTTENLEUTE. Slag Atlas. 2nd Edition. Dusseldorf: Verlag Stahleisen $\mathrm{GmbH}, 1995$. 